\title{
Trachealkanülenmanagement in der neurologisch-neurochirurgischen Frührehabilitation
}

Neurol Rehabil 2016; 22(3): 217-230

(C) Hippocampus Verlag 2016

DOI 10.14624/NR150816.005

\author{
R. Linden, D. A. Nowak, M. Ponfick
}

\section{Zusammenfassung}

Das Entwöhnen von der Beatmung und das Abtrainieren von der Trachealkanüle haben sich zu Behandlungsschwerpunkten in der neurologisch-neurochirurgischen Frührehabilitation (NNFR) entwickelt und stellen hohe Anforderungen an die Kompetenz und die Zusammenarbeit im therapeutischen Team. Trotz des Fehlens einer breiten Evidenzbasis für einen großen Teil der Behandlungsmaßnahmen stellt die vorliegende Arbeit ein standardisiertes, erfahrungsbasiertes, klinisch-pragmatisches Vorgehen für die Entwöhnung von der Trachealkanüle vor.

Schlüsselwörter: Trachealkanüle, Dysphagie, Dekanülierung, Atmung

HELIOS Klinik Kipfenberg

\section{Einleitung}

Die immer frühzeitigere Verlegung von neurologisch Kranken aus der intensivmedizinischen Akutbehandlung in die neurologisch-neurochirurgische Frührehabilitation (NNFR) führt $\mathrm{zu}$ einer stetig wachsenden Zahl von tracheotomierten Patienten in Einrichtungen der neurologischen Frührehabilitation. 42\% der in die Frührehabilitation aufgenommenen Patienten sind mit einem absaugpflichtigen Tracheostoma versorgt, 24\% davon werden invasiv über eine Trachealkanüle (TK) beatmet, wie Ergebnisse einer multizentrischen Erfassung aus dem Jahr 2014 belegen [55]. Hauptindikation für eine Tracheotomie ist die voraussichtliche Langzeitbeatmung aufgrund von internistischen bzw. neurologischen Erkrankungen sowie schwere Dysphagien mit hochgradiger Speichelaspirationsgefahr. In den letzten Jahren nimmt in der NNFR vor allem die Zahl der Patienten mit einer sogenannten Critical-Illness-Polyneuropathie (CIP) zu [50, 57]. Speziell diese Patientengruppe benötigt lange invasive Beatmungszeiten, und die Respiratorentwöhnung gestaltet sich oft schwierig [50, 56, 57]. Hieraus ergeben sich neue Herausforderungen an das Behandlungsteam bestehend aus Ärzten, Therapeuten und Pflegekräften [51].

Die Versorgung mit einer TK bedeutet für den Patienten einen einschneidenden Eingriff, da hierdurch stimmhaftes Sprechen, Riechen, effektive Reinigungsfunktionen wie Husten aufgehoben und die Atmung sowie auch das Schlucken maßgeblich verändert sind $[4,7,68]$. Die vordergründigen Behandlungsziele in der Frührehabilitation sind daher eine schnellstmögliche Entwöhnung vom Respirator sowie von der TK unter maximaler Sicherheit des Patienten. Das schrittweise
Entwöhnen von der TK in Verbindung mit der Restitution dysphagischer Symptome unter Vermeidung von Sekundärkomplikationen wie Infektionen der Atemwege zwingen zu einer engen Zusammenarbeit von Pflege, Therapeuten und Ärzten. Die Entlassung mit TK aus der NNFR steigert das Sterberisiko in der außerklinischen Weiterversorgung erheblich [2, 55]. Innerhalb eines Jahres sterben bis zu 50\% der Patienten, die mit TK aus der NNFR entlassen wurden, von den ohne Trachealkanüle Entlassenen lediglich 15\% [50]. Nicht zuletzt auch aus volkswirtschaftlicher Sicht ist eine hohe Erfolgsquote bei der Entwöhnung von der invasiven Beatmung und TK anzustreben, da den Kostenträgern nach § 37 SGB V für die außerklinische Weiterversorgung von Tracheotomierten und eventuell auch beatmungspflichtigen Patienten sehr hohe Kosten entstehen. Es wurde nachgewiesen, dass durch das Implementieren eines multidisziplinären TK-Managements eine frühzeitigere Dekanülierung und im Zusammenhang damit auch eine funktionelle Verbesserung in den Alltagsleistungen erzielt werden kann $[14,25,51]$.

\section{Tracheostoma-Anlageformen}

In der Akutversorgung erfolgt zur Sicherung der Atmung in der Regel eine oropharyngeale Intubation. Entsprechend den Leitlinien des prolongierten Weanings sollte nach 14-21 Tagen invasiver Beatmung eine Tracheotomie erfolgen [59, 61]; besteht eine anhaltend hochgradige Speichelaspirationsgefahr, sollte möglichst frühzeitig innerhalb der ersten 4-7 Tage eine Tracheotomie zur Vermeidung von Larynxschäden durchgeführt werden [1, 9, 11, 29, 38, 74, 73]. Eine signifikante Reduktion der Pneumonierate, der Beatmungsdauer, des Intensivaufenthal- 


\section{Management of tracheal cannula in early neurological and neurosurgical rehabilitation}

R. Linden, D. A. Nowak, M. Ponfick

\section{Abstract}

Weaning from assisted ventilation and tracheal cannula after tracheotomy are integral parts of early neurological rehabilitation and require a high level of clinical experience and practical knowledge within the therapeutic team. Despite the fact that evidence-based treatment strategies do not exist, the authors present a standardized, pragmatic, experience-based approach for weaning from assisted ventilation and tracheal cannula in neurological rehabilitation.

Keywords: tracheal cannula, dysphagia, decannulation, ventilation

Neurol Rehabil 2016; 22(3): 217-230

(C) Hippocampus Verlag 2016

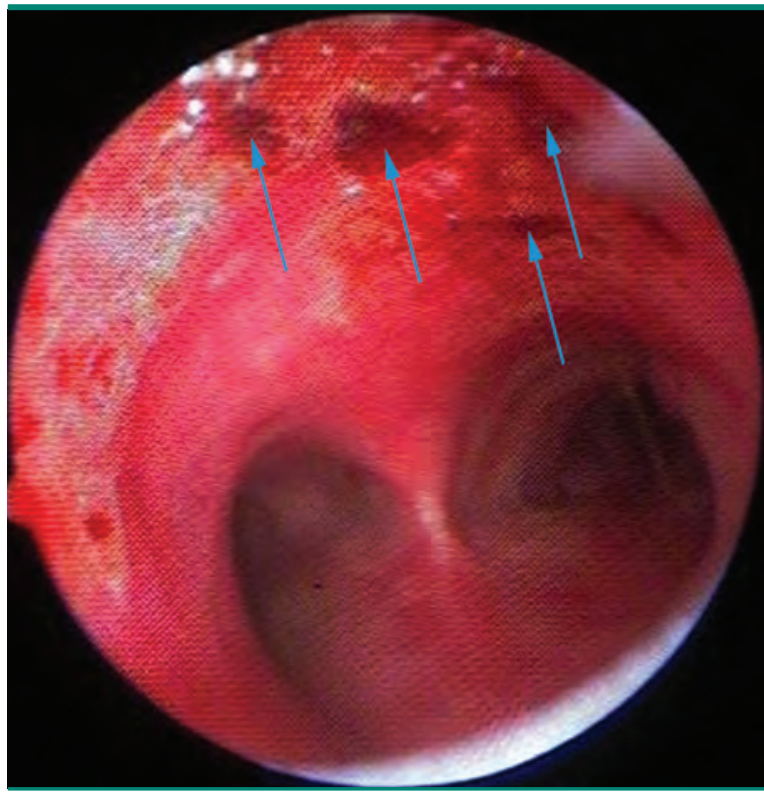

Abb. 1: Absaugtraumen (blaue Pfeile) distal des TK-Lumens

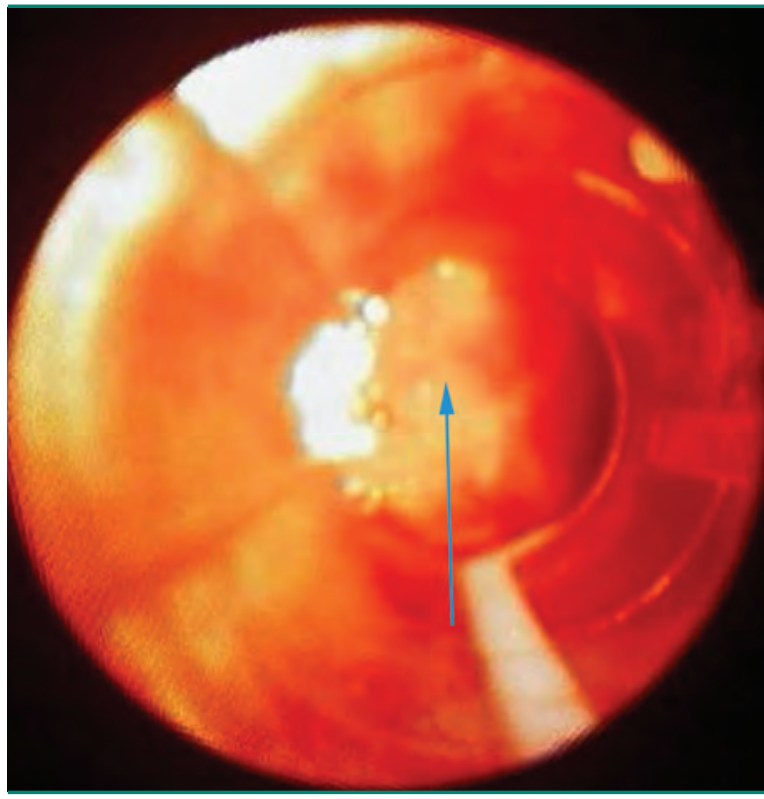

Abb. 2: Vorwölbung der Pars membranacea (blauer Pfeil) und partielle Verlegung der TK-Öffnung tes und/oder der Mortalität durch eine Frühtracheotomie (4.-7. Tag nach Intubation) konnte bisher keine Studie nachweisen $[61,70]$. Je nach Indikation erfolgt eine dauerhafte, plastisch angelegte oder eine temporäre Tracheostomie in Höhe des zweiten bis dritten Trachealknorpels [19]. Bei ersterer wird ein gestielter Lappen der Trachealvorderwand am unteren Wundpol der Halshaut angenäht und die übrigen Hautränder zirkulär an der Trachea fixiert. Bei den temporären, dilatativen Tracheotomien haben sich folgende Methoden etabliert:

- Mehrschritt-Bougier-Verfahren nach Ciaglia mit in der Größe ansteigenden konischen Dilatatoren [10],

- das neuere Einschritt-Bougier-Verfahren mit einem einzigen konisch verlaufenden Dilatator [6],

- das Zangenverfahren nach Griggs [28],

- die retrograde translaryngeale Tracheotomie nach Fantoni [21],

- das Percu-Twist-Verfahren von Fovra und Quintel mit einem Schraubdilatator mit selbst schneidendem Gewinde [26].

Bei allen Anlagetechniken ist die mittige und horizontale Anlage des Stomakanals für die korrekte Lage der TK und zur Vermeidung von Sekundärschäden entscheidend.

\section{Komplikationen}

Auf den rehabilitativen Verlauf wirken sich vor allem Spätkomplikationen wie Stenosen und Tracheomalazien, die nicht selten erst nach Dekanülierung manifest werden, sowie die Bildung von die Atmung beeinträchtigenden Granulationen und Granulomen aus [20, 22, 27, 45, 66]. Arrosionsblutungen werden meist durch intratracheales Absaugen hervorgerufen und weisen häufig auf eine Fehllage der TK hin (Abb. 1).

Auftretende Atemnot ist oft die Folge einer Verborkung der TK und sollte durch einen raschen TK-Wechsel behoben werden. Bei ausbleibender Besserung ist eine endoskopische Ursachenabklärung zwingend erforderlich. Bei Fehllage der TK kann die Vorwölbung der Pars membranacea den Atemweg verlegen und das Abhusten von Sekret beeinträchtigen (Abb. 2).

Vor der Entlassung in die häusliche Intensivpflege oder in eine Weiterversorgungseinrichtung ist eine Epithelialisierung des Tracheostomas zu empfehlen [49, 61]. Eine Zusammenfassung der Früh- und Spätkomplikationen ist in Tabelle 1 dargestellt.

\section{Kanülentypen}

Mittlerweile gibt es eine große Palette an verschiedenen TK-Typen auf dem Markt, die sich in Bauart, Material, Form und Funktion unterscheiden [33, 34]. Grundsätzlich kann zwischen blockbaren (mit Cuff) und unblockbaren, einlumigen und doppellumigen (mit Innenkanüle), gefensterten und ungefensterten TK unterschieden 


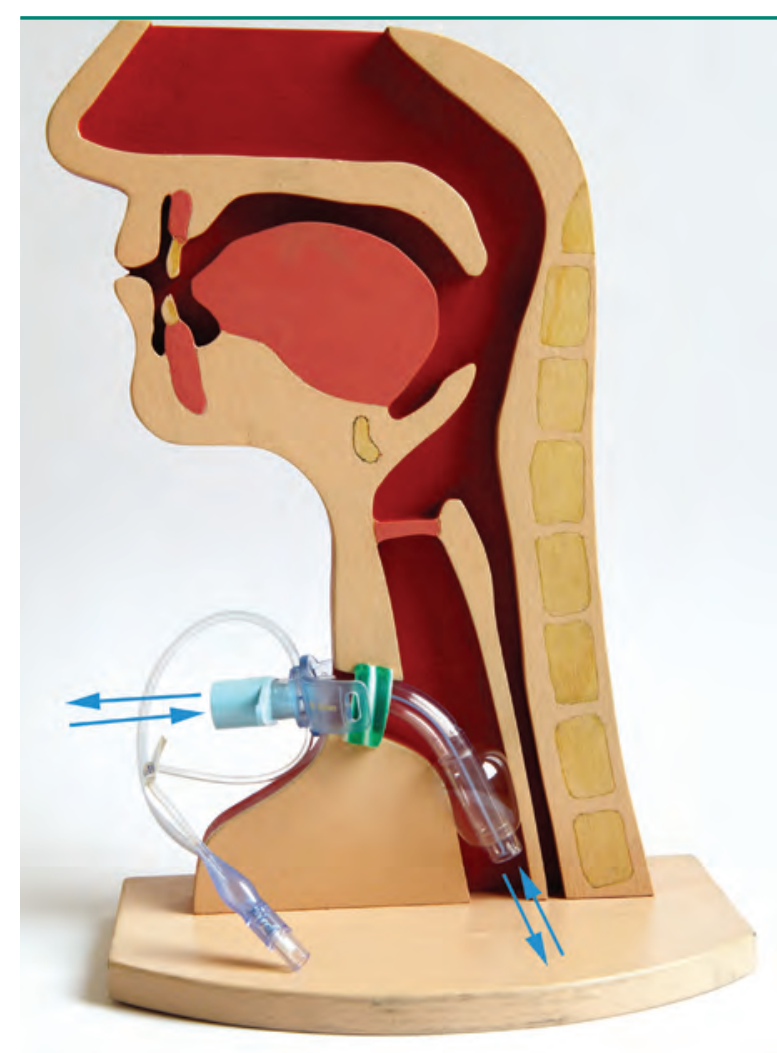

Abb. 3: Luftstrom (blaue Pfeile) bei geblockter TK

Tab. 1: Früh- und Spätkomplikationen bei TK-Versorgung

\begin{tabular}{l|l}
\hline Frühkomplikationen & Spätkomplikationen \\
\hline Blutung & Tracheomalazie \\
\hline Weichteilinfektion & Trachealstenose \\
\hline Mediastinalemphysem & $\begin{array}{l}\text { Schleimhautmetaplasie mit nachfol- } \\
\text { gender Granulombildung }\end{array}$ \\
\hline frakturierte Knorpelspange & Dislokation der Kanüle \\
\hline tracheoösophageale Fistel & $\begin{array}{l}\text { Herniation von Trachealschleimhaut } \\
\text { bei gesiebten Kanülen (Abb. 9) }\end{array}$ \\
\hline & Verborkung der Schleimhaut \\
\hline & späte tracheoösophageale Fistel \\
\hline
\end{tabular}

werden. TK gibt es in verschiedenen Längen und Größen, wobei sich die Größenangabe auf den Innen- und Außendurchmesser bezieht. Fast alle Kanülen sind mit einem 14-mm-Normkonnektor ausgestattet, der verschiedene Aufsätze wie Beatmungssysteme, künstliche Nasen oder Sprechventile erlaubt. Die Änderungen des Luftstromes je nach Zustand der Blockung der TK sind in Abbildung 3 und 4 angegeben.

\section{TK mit Cuff (blockbare Kanülen)}

Blockbare TK haben am distalen Ende einen »high volume, low pressure cuff«. Zur Vermeidung von Druck-

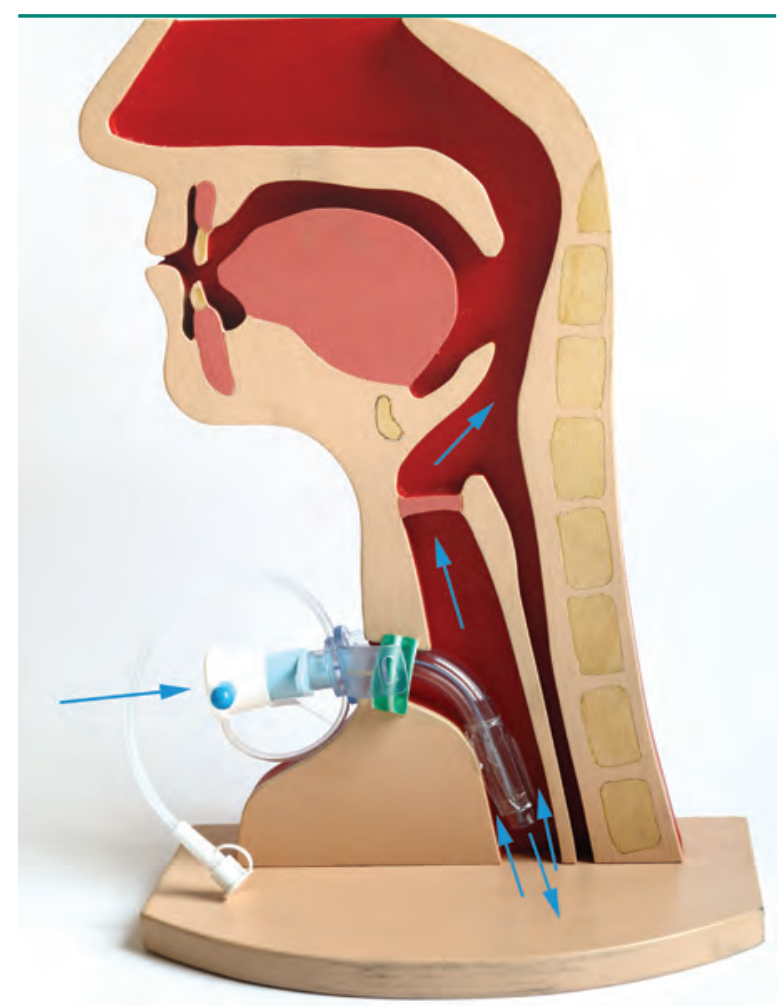

Abb. 4: Luftstrom (blaue Pfeile) bei entblocktem Cuff

schäden darf der Cuff-Innendruck nicht 25 mmHG bzw. $35 \mathrm{cmH}_{2} \mathrm{O}$ überschreiten [47]. Durch den Cuff wird die Luftröhre nach oben hin abgedichtet und ermöglicht somit eine maschinelle Beatmung in der Regel ohne Luftverlust. Sie bietet hierdurch auch einen Schutz vor dem Eindringen von z. B. aspiriertem Speichel in die tieferen Atemwege. Der Cuff unterscheidet sich je nach TK-Typ in seiner Form und seinem Material. So gibt es zylindrische, eiförmige und konische Formvarianten, im entblockten Zustand mehr oder weniger stark faltenbildende oder eng anliegende Cuffs (SealGuard-Konzept von Covidien ${ }^{\mathrm{TM}}$ ) (Abb. 5). Letztere sind nicht nur für einen schonenden TK-Wechsel (vor allem bei dilatativen Tracheostomata) von Vorteil, sondern begünstigen auch entscheidend das methodische Vorgehen in der TK-Entwöhnung.

\section{TK mit Cuff und Fensterung oder Siebung}

Gefensterte bzw. gesiebte TK werden irreführenderweise oft als Sprechkanüle bezeichnet, ermöglichen sie doch nicht per se das Sprechen. Sie haben eine große Öffnung (Fenster) oder mehrere kleine Öffnungen (Siebung) im Bereich der Krümmung. Sie besitzen jeweils zwei Innenkanülen, eine geschlossene, d.h. ohne Öffnungen, und eine gefensterte oder gesiebte (Abb. 6). 


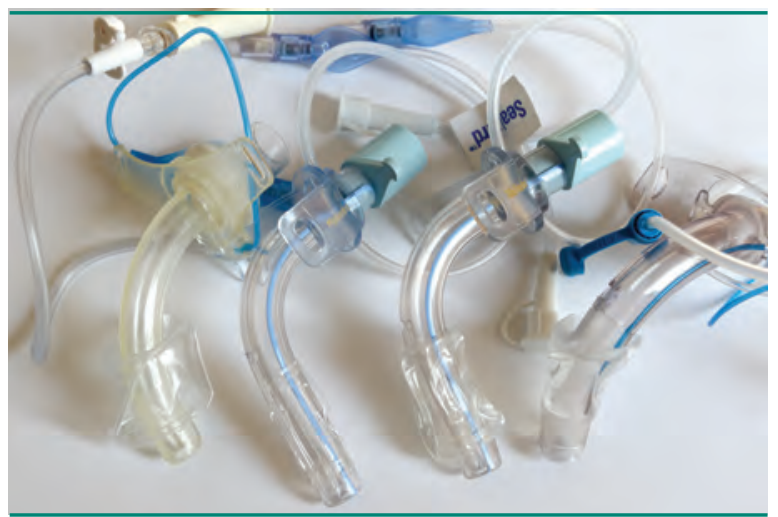

Abb. 5: Verschiedene blockbare TK mit verschiedene Cuffs (2. von links eng anliegender Cuff SealGuard)

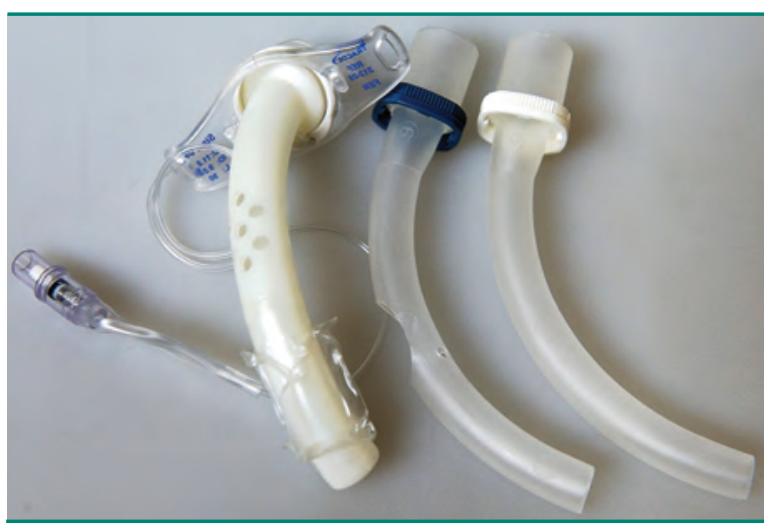

Abb. 6: Gesiebte TK mit Innenkanülen

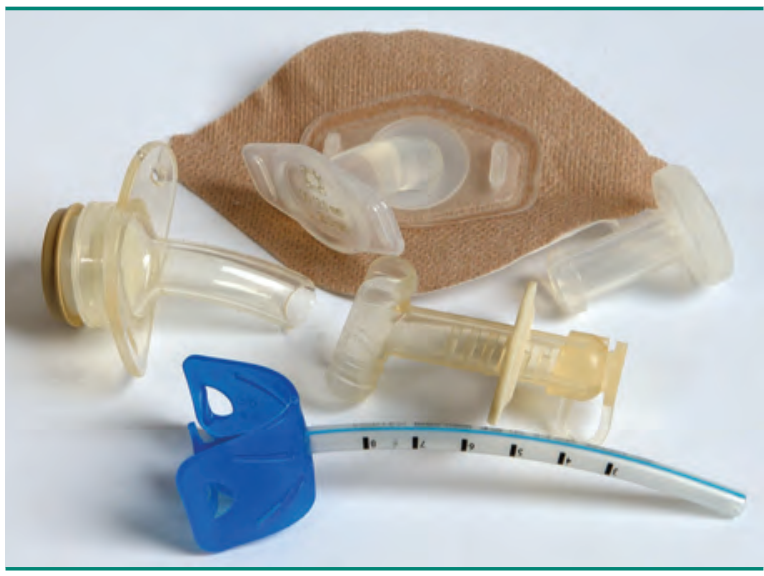

Abb. 7: Verschiedene Platzhalter, Kurzkanüle und Mini-TK

Bei Verwendung der geschlossenen Innenkanüle erfüllt sie die gleiche Funktion wie eine einwandig blockbare TK. Wird die genfensterte/gesiebte Innenkanüle eingesetzt, ermöglicht sie bei optimaler Platzierung und Verwendung eines Sprechventils das stimmhafte Sprechen. Voraussetzung ist die korrekte Lage der Öffnungen im Trachealkanal, was stets durch eine endoskopische Lagekontrolle bestätigt werden sollte. Üblicherweise werden diese Kanülen zum Sprechen zusätzlich ent- blockt, um das funktionelle Tracheallumen zu erweitern. Eine regelmäßige Reinigung der Innenkanüle verhindert die Verlegung der TK durch Sekret, was eine längere Liegedauer ermöglicht.

\section{TK mit subglottischer Absaugung}

Ein zweiter innenwandig oder außenwandig an der TK verlaufender Schlauch, der in einer kleinen Öffnung oberhalb des Cuffs endet, ermöglicht das Absaugen von auf dem Cuff sitzendem Sekret. Ursprünglich diente der Schlauch einer externen Luftzufuhr via Glottis und ermöglichte eine (künstliche) Phonation bei geblockter TK [37]. Durch die Möglichkeit der subglottischen Sekretdrainage kann die Inzidenz einer Aspirationspneumonie signifikant reduziert werden [36]. Seit Einordnung der TK mit subglottischer Absaugung in die Empfehlungskategorie IA des Robert-Koch-Instituts im Oktober 2013 zur Prävention von Respirator-assoziierten Pneumonien etablierte sie sich in zunehmendem Maße in den klinischen Alltag [1]. Je nach Sekretbeschaffenheit oder Lage der Öffnung kann das subglottische Absaugen erschwert sein oder gar unmöglich werden. Hier hilft meist eine kurze manuelle Lageveränderung der Trachealkanüle oder Veränderung der Kopfstellung. Vor den häufig empfohlenen subglottischen Spülungen mit Kochsalzlösungen oder sonstigen Flüssigkeiten wird eindringlich gewarnt, da gerade Flüssigkeit über sogenannte Aspirationsstraßen am Cuff vorbei in die tieferen Atemwege vordringen kann [3].

\section{TK ohne Cuff (unblockbare TK)}

TK ohne Cuff werden nach abgeschlossenem Weaning und Ausschluss einer Aspiration bei noch bestehender trachealer Absaugnotwendigkeit oder Obstruktionen im Kehlkopfbereich eingewechselt. Auch hier gibt es TK mit und ohne Innenkanüle, je nach Bedarf auch mit Fensterung oder Siebung.

\section{Platzhalter}

Sofern die Dekanülierung noch mit Unsicherheiten verbunden ist, kann zur Sicherung des Stomakanals vorübergehend ein sogenannter Platzhalter eingewechselt werden [23]. Nachteil vieler gängiger Platzhalter ist das schwierige Einlegen insbesondere bei dilatativen Tracheostomata und/oder auch die Gefahr der Dislokation. Alternativ kann eine Kurzkanüle aus Silikon oder eine sogenannte Mini-TK eingesetzt werden, die im Tracheallumen zum Liegen kommt (Abb. 7).

\section{Wahl der Kanüle aus therapeutischer Sicht}

Nicht selten knüpfen die unterschiedlichen Berufsgruppen in der klinischen Praxis ganz unterschiedliche 
Zielsetzungen an die Wahl der TK. Leider sind den Verantwortlichen nicht immer die Vor- und Nachteile der einzelnen TK ausreichend geläufig. Die Abdichtung der Trachea durch den Cuff wird durch ihre Form bestimmt. Da der Trachealquerschnitt in bis zu 50\% der Fälle nicht kreisrund ist (Abb. 8), kann bei beatmeten Patienten Luft am Cuff vorbei nach oben entweichen, sodass ein sogenanntes leaking entsteht. Über diese undichten Stellen (Aspirationsstraßen) kann auch Sekret in tiefere Areale vordringen, eine der Hauptursachen für nosokomiale Pneumonien. Die immer noch verbreitete Denkweise, dass ein über das Normalniveau hinausgehendes Aufblocken des Cuffs zu einer besseren Abdichtung führt, erhöht lediglich die Gefahr von Drucknekrosen der Trachealschleimhaut und unter Umständen nachfolgender Stenosierungen und sollte daher vermieden werden [46]. Entscheidend für die optimale Wahl einer TK sind sowohl der Innen- als auch der Außendurchmesser. Der Innendurchmesser bestimmt den Atemwegswiderstand im geblockten Zustand und sollte, um das Weaning nicht zu beeinträchtigen, nicht zu klein ausfallen. Der Außendurchmesser ist entscheidend für den Raum zwischen TK und Trachea über den bei Sprechventil- oder Verschlusskappenaufsatz (SV/VK-A) der Luftaustausch via Glottis erfolgt. Daher sollte der Außendurchmesser möglichst klein gehalten werden. Je nach Hersteller zeigen vergleichbare TK bei gleichem Innendurchmesser Unterschiede in der Wandstärke: $1 \mathrm{~mm}$ (einwandige TK) bis $\mathrm{zu} 3 \mathrm{~mm}$ (TK mit Innenkanüle) [48]. Dünnwandige TK sind materialbedingt meist starrer. Die Angabe des Außendurchmessers berücksichtigt jedoch nicht den Cuff im entblockten Zustand, der je nach Material und Form das funktionelle Tracheallumen mehr oder weniger stark einschränkt. Hier hat ein eng anliegender Cuff entscheidende Vorteile, da er den TK-Wechsel, insbesondere bei einem nicht epithelialisierten Dilatationstracheostoma, weit schonender ermöglicht.

Das verbleibende funktionelle Lumen bei einer eingesetzten TK mit SV/VK-A wird bestimmt durch die Anatomie und den Durchmesser der Trachea, der beim Mann durchschnittlich 18,7-20,8 mm, bei der Frau 15,7$18,5 \mathrm{~mm}$ beträgt [5]. Bei der Entscheidung für eine TK ist daher das Verhältnis zwischen Außendurchmesser der TK und dem Durchmesser der Trachea entscheidend. Da letzterer gewöhnlich nicht bestimmt wird, ist die Beurteilung eines ausreichenden Lumens klinisch nur indirekt über die Beurteilung der Atmung oder mittels analoger/digitaler Messung des Expirationsdrucks möglich [39]. Wie Untersuchungen an Patienten mit Tracheastenosen gezeigt haben, zeigt sich erst bei einer Einengung des Trachealumens über $70 \%$ klinisch eine Belastungsdyspnoe, die sich durch einen inspiratorischen Stridor bemerkbar machen kann [12, 44]. Sollte eine anhaltend erschwerte Atmung trotz Wechsel auf eine kleinlumigere TK fortbestehen, empfiehlt sich die endoskopische Abklärung.

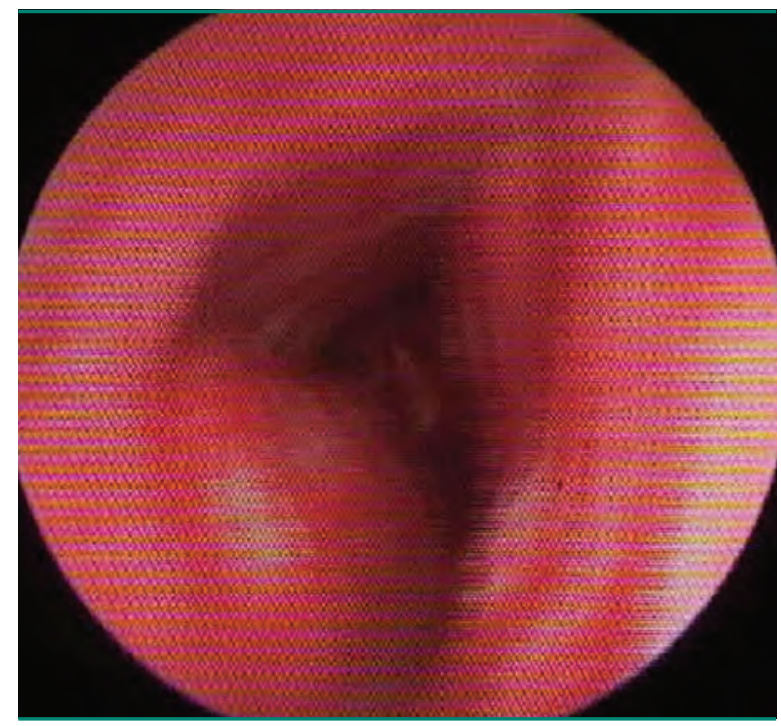

Abb. 8: Dreispitzförmiger Trachealquerschnitt

Zur Erweiterung des funktionellen Tracheallumens unter SV/VK-A kann eine gefensterte TK verwendet werden, was in der Regel mit einer Zunahme des Außendurchmessers verbunden ist. Die Summe der Öffnungen der Innenkanüle, die in der Regel größer ist als das tatsächliche TK-Lumen, reicht bei korrekter Lage der Öffnungen für eine suffiziente Respiration bei Verschluss der TK aus. Liegt jedoch ein Teil der Öffnungen nicht unmittelbar im Trachealkanal, kommt es zu einer Einschränkung bis hin zu einer kompletten Verlegung der Atemwege. Ebenso kann bei zäher Sekretproduktion das hochgehustete Sekret die Öffnungen verlegen und somit die Atmung beeinträchtigen. Durch einen regelmäßigen Wechsel der Innenkanüle kann dies verhindert werden. Liegt ein Teil der Öffnungen direkt der Trachealschleimhaut an, kann sich diese durch die Öffnungen ins Tracheallumen hineinwölben, was (insbesondere nach längerem Einliegen der gefensterten Innenkanüle) bei einem Wechsel durch Abscherbewegungen zu stark blutenden Schleimhautverletzungen führen kann (Abb. 9).

Generell darf sich die Größe der TK aus den oben genannten Gründen nicht an der Größe des Tracheostomakanals orientieren, was in der klinischen Praxis zur Vermeidung von Sekretaustritt aus einem zu großen Tracheostoma leider noch oft der Fall ist. Tracheale Engstellen unterhalb der TK können zur Sicherung der Atmung durch eine extralange TK überbrückt/geschient werden. Die nicht korrekte Lage einer TK kann unter Umständen durch das Einwechseln einer Kanüle mit einem anderen Krümmungswinkel behoben werden. Inkorrekte TK-Lagen sind meist durch hartnäckigen Husten, Atemprobleme oder ein blutiges Absaugsekret infolge von Absaugtraumen klinisch auffällig. Zur Reduzierung des Risikos für eine Aspirationspneumonie sollte, wie bereits oben beschrieben, generell eine Kanüle mit subglottischer Absaugung gewählt werden $[1,8,13,30,36]$. 


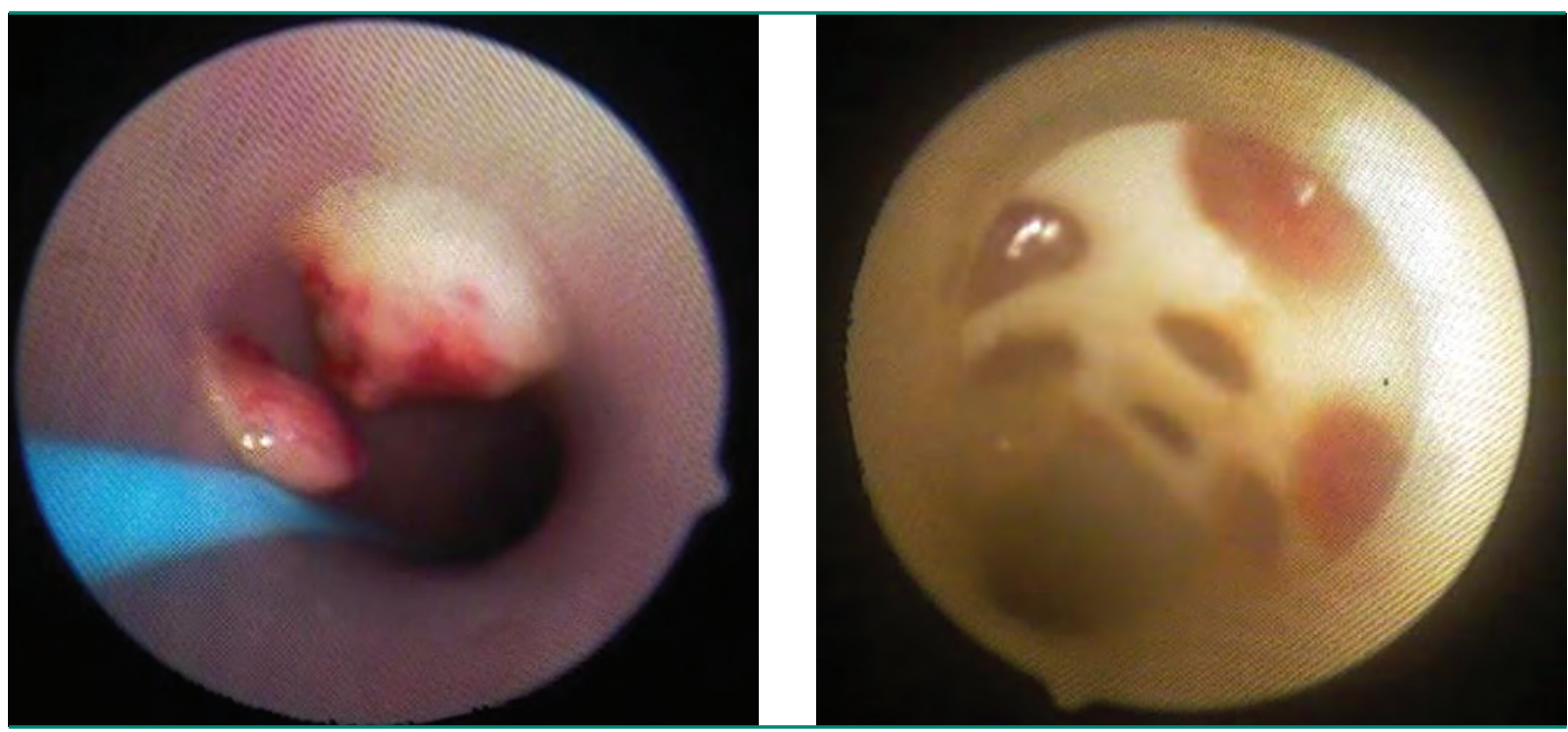

Abb. 9: Trachealschleimhaut drückt sich durch die Öffnungen einer gefensterten TK mit Innenkanüle

\section{TK-Wechsel}

Richtlinien oder Empfehlungen hinsichtlich der Liegezeit einer TK existieren bislang nicht. Die Hersteller geben keine konkreten Zeitintervalle vor, in denen eine Kanüle gewechselt werden sollte. In der NNFR variieren die Expertenempfehlungen zwischen täglichem Wechseln bis hin zum Wechseln in dreiwöchigen Abständen, abhängig auch von der Anlageform des Tracheostomas. Ein häufiger Wechsel in kurzen Zeitabständen birgt bei dilatativen Tracheostomata die Gefahr von Verletzungen im Bereich des Stomakanals und der Trachea. Eine verborkte Trachealkanüle muss umgehend ausgewechselt werden. Lediglich bei neuangelegten Tracheostomata sollte ein Wechsel innerhalb der ersten fünf Tage nach Anlage aufgrund der erhöhten Gewebsvulnerabilität und -instabilität unterlassen werden [61]. Ist dies dennoch notwendig, empfiehlt sich der Wechsel in Intubationsbereitschaft [61].

\section{SV/VK-A}

Das schrittweise Entwöhnen von der TK erfolgt unter kontrollierten Bedingungen durch temporäres Entblocken der TK und Aufsetzen eines SV/VK-A, was dem Patienten das stimmhafte Sprechen ermöglicht. Beim Aufsetzen eines SV muss eine effiziente Exspiration, bei der VK-A auch eine effiziente Inspiration gewährleistet sein. Wie bereits erwähnt, hängt dies entscheidend von der Anatomie der Trachea und den verschiedenen TKEigenschaften ab. Beim SV, einem Einlassventil, wird die Atemluft über die Kanüle eingeatmet und durch Verschluss des Ventils über die Trachea ausgeatmet. Sowohl die VK-A als auch das SV ermöglichen neben der Phonation eine effektivere willkürliche und reaktive pharyngolaryngeale Reinigung sowie durch den Glottisschluss eine effektive broncho-tracheale und laryngopharyngeale Sekret-Clearance [32, 58]. Nachteil des SV ist das raschere Austrocknen der Trachealschleimhaut durch das fehlende Anfeuchten und Erwärmen der Inspirationsluft. Das macht die Trachealschleimhaut vulnerabler für Absaugtraumata und Infektionen. Bei Patienten mit chronisch obstruktiver Lungenerkrankung (COPD) besteht auch die Gefahr einer Überblähung der Lunge bei unzureichender Exspiration durch ein sogenanntes "Airtrapping « [6o].

Ein wesentlicher Vorteil der VK-A ist die zusätzliche inspiratorisch verbesserte pharyngolaryngeale Reizverarbeitung, wodurch Speichelresiduen im Einatemstrom eher gespürt werden können. Weitere Vorteile sind die atemstimulierende und atemvertiefende Wirkung, wodurch die Atemmuskulatur trainiert wird, und die wegen des natürlichen Atemweges verminderte Austrocknung der Trachealschleimhaut [39]. Die Verwendung der VK-A bedeutet aber auch eine vermehrte inspiratorische Atemarbeit, was zu einer rascheren Atemermüdung führen kann. Die Auswahl des TK-Aufsatzes setzt sowohl ein gewisses Maß an Erfahrung als auch eine engmaschige Kontrolle und Überwachung der Atmung und des Speichelschluckaktes voraus. So muss die Toleranz des Aufsatzes in allen Ausgangsstellungen klinisch geprüft werden, um lagerungsabhängige Atembeeinträchtigungen auszuschließen. Es ist zu betonen, dass im Falle einer SV/VK-A-Versorgung eine Entblockung des Cuffs unerlässlich ist, da ansonsten die Erstickung des Patienten droht, weshalb nur erfahrenes Personal die Versorgung mit SV/VK-A übernehmen sollte.

Auch bei invasiv beatmeten Patienten kann in Abhängigkeit der Aspirationsgefahr nach Aufheben der TK-Blockung temporär ebenfalls ein Sprechventil (Passy- 
Muir-Ventil) (Abb. 10) eingesetzt werden [40, 52, 53]. Voraussetzung ist auch hier ein ausrechendes funktionelles Tracheallumen zwischen TK-Außendurchmesser und Trachea und minimaler Exspirationsdruck seitens des Patienten. Durch das Entblocken entsteht im sonst geschlossenen Beatmungssystem eine Leckage, was durch eine Anpassung der Beatmungsparameter ausgeglichen werden muss. Eine Kontraindikation für diese Maßnahme ist ein erhöhter positiver endexspiratorischer Druck (PEEP) (> 5 mbar). Alternativ zum Pass-Muir-Ventil kann auch nach Aufhebung der TK-Blockung auf den Einsatz eines SV verzichtet werden, was dann sowohl exspiratorisches als auch inspiratorisches Sprechen ermöglicht. Bei Patienten mit vollständiger Atemlähmung kann ggf. auf das SV verzichtet werden, dennoch ist inspiratorisch ein stimmhaftes Sprechen möglich, was die Lebensqualität erheblich verbessern kann. Die Alarmgrenzen und je nach Beatmungsgerät auch weitere Beatmungsparameter müssen geändert werden und eine Speichelaspirationsgefahr ausgeschlossen sein.

\section{Auswirkungen der TK auf das Schlucken}

Die Frage, inwieweit eine geblockte TK den Schluckakt beeinträchtigt, wurde in der Vergangenheit sehr kontrovers diskutiert. Unterschiedliche Studiendesigns, die Verwendung verschiedener Untersuchungsmethoden und heterogene Patientenkollektive erschweren den direkten Vergleich zwischen den Studien. Einige Autoren konstatieren einen negativen Einfluss der geblockten gegenüber der ungeblockten Kanüle auf die Biomechanik des Schluckaktes [4, 17, 40]. So zeigen sich Einschränkungen in der laryngealen als auch hyolaryngealen Exkursion verbunden mit einer reduzierten Ösophagussphinkteröffnung bzw. einer veränderten pharyngealen Kontraktion [40]. Andere Autoren konnten keinen Einfluss der TK auf das Schlucken belegen [18, 41, 42, 69]. Die Hypothese, dass durch den Verschluss der TK die Aspirationsgefahr reduziert werden kann, wurde bislang nur bei dysphagischen Patienten mit Tumoren der Kopf- und Halsregion berichtet [16, 54, 67], wobei auch hier gegenteilige Ergebnisse vorliegen [43]. Bei geblockter TK kommt es nachweislich zu einer Reduktion der Schluckfrequenz, was auf die Reizdeprivation des pharyngolaryngealen Raums durch den umgeleiteten Atemstrom zurückzurückzuführen ist [64]. Die Hypothese, ob dies einen sogenannten »learned non-use« der Schluckmuskulatur und eine Verstärkung der Schluckstörung begünstigen könnte [55], muss in weiteren Untersuchungen bestätigt werden.

\section{Oralisierung bei geblockter TK}

Eine dauerhaft geblockte TK ist nicht per se eine Kontraindikation für die Oralisierung. Kontinuierlich beatmete Patienten wie z.B. Muskelerkrankte oder solche mit

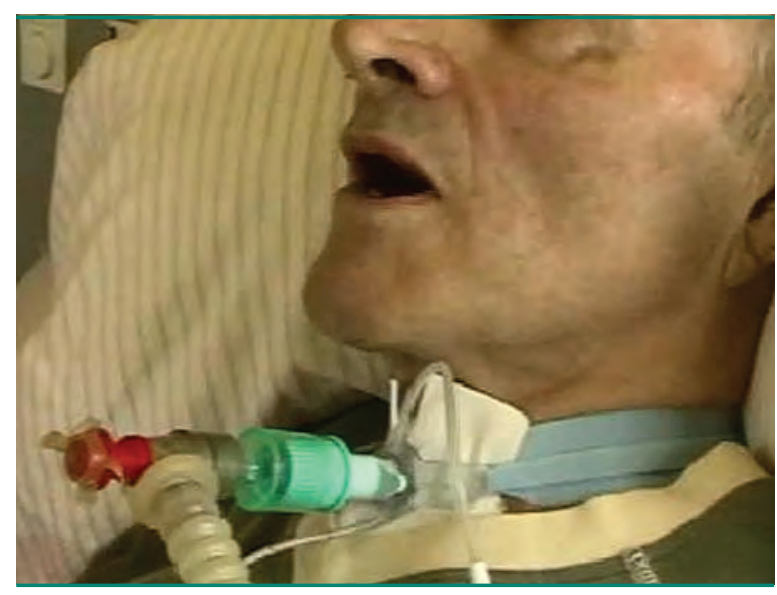

Abb. 10: Passy-Muir-Ventil, das stimmhaftes Sprechen während invasiver Beatmung ermöglicht

einem hohen Querschnittsyndrom ohne Schluckstörung können ohne nachweisbare Aspiration mit geblockter TK essen und trinken. Voraussetzungen für die Verabreichung von Flüssigkeiten und fester Kost (mit oder ohne Konsistenzeinschränkungen) sind neben ungestörten kognitiven Leistungen (u.a. Aufmerksamkeit und Konzentration) eine differenzierte klinische Abklärung der Schluckqualität, gestützt durch bildgebende Untersuchungstechniken während der Nahrungsaufnahme.

\section{Mundpflege}

Die Mundhöhle gehört zu den mikrobiologisch am dichtesten besiedelten Körperregionen mit 107-1.012 Mikroorganismen pro ml Speichel [63]. Bei 115 tracheotomierten und mit geblockter TK versorgten Patienten mit neurologischen Erkrankungen unterschiedlicher Ätiologie, die auf der Intensivstation einer neurologischen Universitätsklinik behandelt wurden, zeigte sich bereits 5 Tage nach der Tracheostomaanlage bei 36\% eine Verschiebung des physiologischen grampositiven Bakterienspektrums des Oropharynx in den gramnegativen Bereich [2]. Bei der Pathogenese der Ventilator-assoziierten Pneumonie (VAP) ist die Mikroaspiration von erregerhaltigen Sekreten aus dem Nasen-Rachen-Mundraum nachweislich eine der Hauptursachen [65] (Abb. 11 und 12). Wie bereits dargestellt, kommt es anatomisch bedingt auch bei Einlage einer geblockten TK mit »high volume, low pressure cuff « zur Bildung von Speichelstraßen entlang des Cuffs, über die kontaminiertes Sekret in die tieferen Atemwege gelangen kann [2]. Die Gefahr der Entstehung einer Aspirationspneumonie ist dabei selbst durch eine engmaschige Kontrolle des Cuff-Drucks nicht vermeidbar [2]. Im Gegensatz dazu kann das Pneumonierisiko durch eine gewissenhafte Mundpflege (Zähne und Zunge) mit antiseptischen Substanzen erheblich reduziert werden, weshalb in der Richtlinie zur Vermeidung der VAP die dreimal tägliche Mundspülung mit Octenidin-haltigen Präparaten empfohlen wird $[15,35,62]$. 


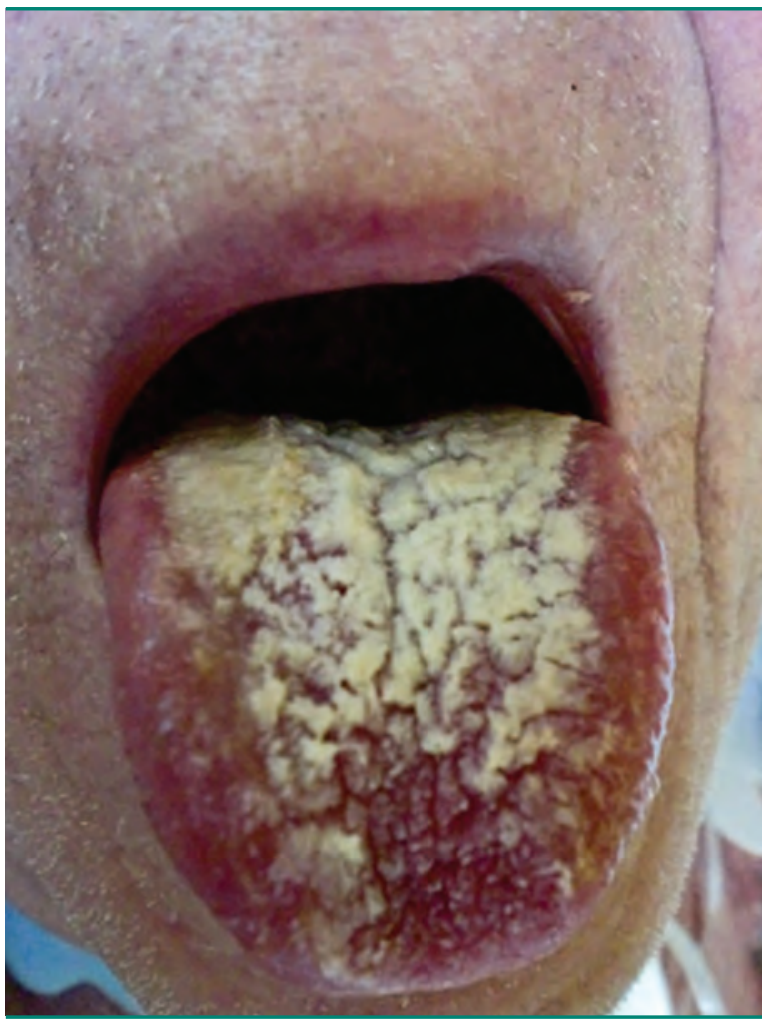

Abb. 11: Zungenbelag, kein Soor

\section{Der Weg zur Dekanülierung}

Für das Abtrainieren der TK gibt es kein allgemeingültiges evidenzbasiertes Ablaufschema. Das therapeutische Vorgehen ist derzeit entscheidend bestimmt durch das Wissen und die Erfahrungen im Umgang mit tracheotomierten, beatmeten und dysphagischen Patienten. Dabei spielt sowohl die Vertrautheit mit den Indikationen und Kontraindikationen der verschiedenen TK-Arten als auch die Interpretation klinischer und bildgebender Schluckuntersuchungsverfahren eine wichtige Rolle. Die für einzelne Erkrankungsentitäten vorliegenden Behandlungsempfehlungen der Dysphagie gründen in der NNFR überwiegend auf Expertenmeinungen [49]. Für die überwiegende Mehrheit der kompensatorischen und restituierenden Behandlungsverfahren fehlen ausreichend große randomisierte Studien, um evidenzbasierte Empfehlungen auszusprechen.

\section{Erste Schritte in Richtung Dekanülierung}

Die physiologische Lenkung des Atemstroms durch Entblocken der TK und des TK-Verschlusses verbessern die Frequenz und Physiologie des Schluckens, das Sekretmanagement und die Atemphysiologie [64]. Weitere schluckstimulierende Verfahren können als vorbereitende Maßnahmen durchgeführt werden [49] (Tab. 2). Grundsätzlich sollte vor jedem Entblocken der TK eine gründliche Mundpflege durchgeführt werden. Die

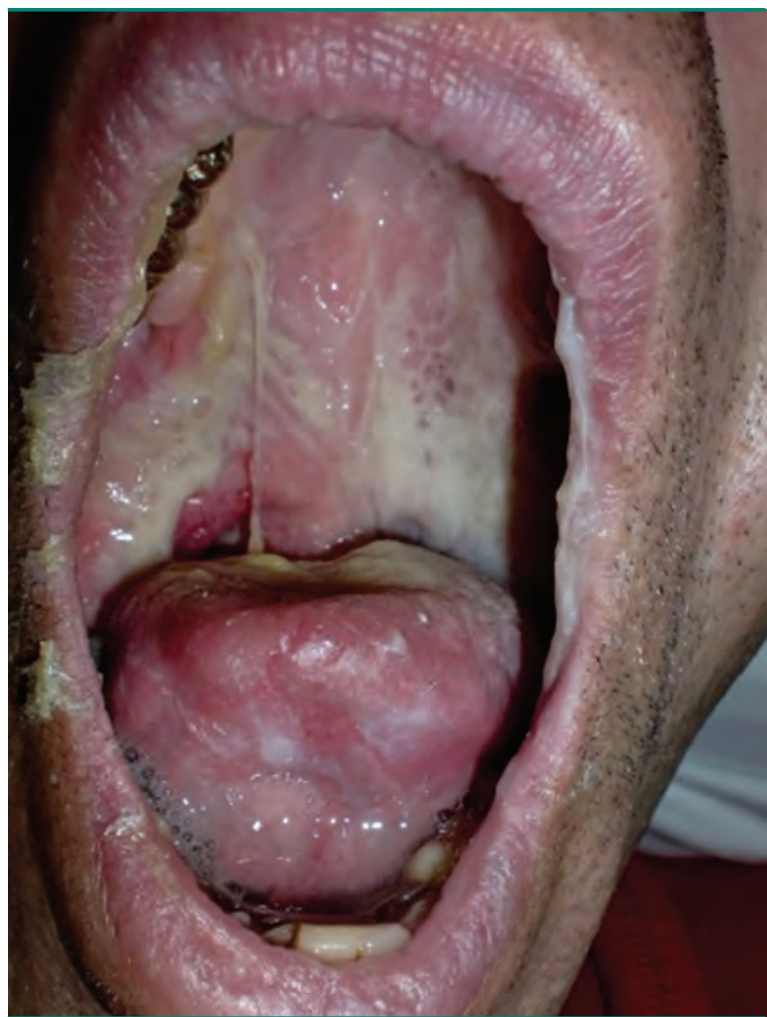

Abb. 12: Sekretbelag am Gaumen

Dauer der ersten Entblockungsphase richtet sich nach den Reaktionen des Patienten auf diese Maßnahme. Der Verschluss der Kanüle sollte erstmalig manuell in der Inspiration erfolgen und anschließend auch in der Exspiration. Unmittelbar einsetzender effizienter Husten mit Anstieg der Schluckfrequenz bei unauffälliger Atmung rechtfertigt eine zeitliche Ausdehnung der Entblockung. In Abhängigkeit von Atemqualität, -anstrengung und -belastbarkeit kann entweder eine SV oder VK-A aufgesetzt werden.

\section{Blauschluck}

Zur qualitativen Beurteilung des Speichelschlucks kann die Zunge mit Patentblau angefärbt werden. Durch intermittierende Absaugkontrollen in zeitlich festgelegten Abständen ergeben sich erste Hinweise zur Aspirationsgefahr. Das Absaugen über eine subglottische Absaugvorrichtung ist weniger invasiv, auch wenn sie bei den meisten Patienten eine Hustenreaktion auslöst. Wegen des Verbleibens des Aufsatzes während der subglottischen Absaugung ist, im Gegensatz zum intratrachealen Absaugen, jederzeit ein (effektiver) Hustenstoß möglich. Ein frühzeitiger subglottischer Farbnachweis deutet auf ein eher hohes Aspirationsrisiko hin, gibt empirisch aber keinerlei Aufschluss über die Menge des Aspirats. Wird kein blaugefärbter Speichel abgesaugt, sollte das nicht generell als Aspirationsausschluss interpretiert werden. Je nach Fließeigenschaft und Menge des Speichels wird 
Tab. 2: Auswahl restituierender und kompensatorischer Therapien zur Behandlung der Schluckstörung nach Schlaganfall in der Frührehabilitation mit Empfehlungsstärken. Modifiziert nach Nowak et al. 2013 [49]

\begin{tabular}{|c|c|c|}
\hline Intervention & ES & Kommentare \\
\hline \multicolumn{3}{|l|}{ restituierende Verfahren } \\
\hline $\begin{array}{l}\text { vorbereitende Stimuli (z. B. streichender Druck, Vibration, Tap- } \\
\text { ping), Mobilisationstechniken (z. B. Widerstandsübungen), } \\
\text { autonome myofunktionelle Übungen }\end{array}$ & A & $\begin{array}{l}\text { Kräftigung des Lippenschlusses und des Wangentonus (u. a. bei fazialer } \\
\text { Parese) zur Verhinderung des Nahrungsaustritts bzw. von Nahrungsretenti- } \\
\text { onen im Wangensulkus sowie des intraoralen Druckverlustes; Kräftigung und } \\
\text { Erweiterung des Bewegungsausmaßes der Zunge zur Sicherung der oralen } \\
\text { Verarbeitung; Verbesserung der Gaumensegelbeweglichkeit zur Verhinderung } \\
\text { der nasalen Penetration }\end{array}$ \\
\hline Üben des Kopfhebens im Liegen (Shaker-Manöver) & A & $\begin{array}{l}\text { Kräftigung der suprahyoidalen Muskulatur und dadurch der Öffnung des obe- } \\
\text { ren Ösophagussphinkters }\end{array}$ \\
\hline $\begin{array}{l}\text { Zunge beim Schlucken zwischen den Schneidezähnen halten } \\
\text { (Masako-Manöver) }\end{array}$ & A & $\begin{array}{l}\text { verstärkte Vorwölbung der Rachenhinterwand, verbesserter Zungenbasis- } \\
\text { Rachen-Abschluss }\end{array}$ \\
\hline elektrische pharyngeale Stimulation & C & keine randomisierten kontrollierten Studien \\
\hline \multicolumn{3}{|l|}{ kompensatorische Verfahren } \\
\hline Kopfneigung nach vorne beim Schlucken (chin tuck) & A & verbesserter Schutz des Kehlkopfeingangs durch Verengung \\
\hline pharyngeale Expektoration & A & $\begin{array}{l}\text { willentliche Rachenreinigung bei pharyngealen Residuen und Vermeidung } \\
\text { einer postdeglutitiven Aspiration }\end{array}$ \\
\hline Kopfdrehung/Kopfkippung zur betroffenen Seite & A & $\begin{array}{l}\text { Abtransport des Bolus über die gesunde Seite, verbesserte Öffnung des obe- } \\
\text { ren Ösophagussphinkters }\end{array}$ \\
\hline supraglottisches Schlucken (SGS) & A & $\begin{array}{l}\text { bewusstes Atemanhalten unmittelbar nach dem Schlucken, dann kurzes } \\
\text { Husten und nochmals leer Schlucken zur Reinigung des Kehlkopfeingangs }\end{array}$ \\
\hline kraftvolles Schlucken & A & Verbesserung des Bolustransportes \\
\hline Mendelsohn-Manöver & A & $\begin{array}{l}\text { Anheben des Larynx und Verlängerung der Öffnung des oberen Ösophagus- } \\
\text { sphinkters }\end{array}$ \\
\hline
\end{tabular}

Empfehlungsstärken (ES): A Hohe Empfehlungsstärke aufgrund starker Evidenz oder bei schwächerer Evidenz aufgrund besonders hoher Versorgungsrelevanz. B Mittlere Empfehlungsstärke aufgrund mittlerer Evidenz oder bei schwacher Evidenz mit hoher Versorgungsrelevanz oder bei starker Evidenz und Einschränkungen der Versorgungsrelevanz. C Niedrige Empfehlungsstärke aufgrund schwächerer Evidenz oder bei höherer Evidenz mit Einschränkungen der Versorgungsrelevanz

eine Aspiration möglicherweise erst verzögert erkennbar. Zudem unterliegen Speichelproduktion, -zusammensetzung und -menge tagesabhängigen Schwankungen bzw. werden durch Medikamente beeinflusst. Eine ausbleibende Schluckaktivität ist per se kein Indikator für ein erhöhtes Aspirationsrisiko, ist sie doch abhängig von der Speichelproduktion, die bei Mundatmern reduziert sein kann. Eine vermehrte Speichelsekretion kann medikamentös vermindert werden. Je nach Verlauf und Belastbarkeit können die beschriebenen Maßnahmen in Abhängigkeit von den individuellen Patientenressourcen als auch den personellen Ressourcen der Klinik mehrmals täglich wiederholt werden. Grundsätzlich sollte die Behandlung engmaschig, wenn möglich täglich erfolgen. Abbruchkriterien sind respiratorisch kritische Situationen, die sich in vegetativen Stresszeichen äußern können, bzw. ein eindeutiger Aspirationsnachweis. Ursachen und Erklärungen für etwaige Auffälligkeiten müssen gezielt gesucht und benannt werden.

Repetitiver postdeglutitiver trockener Husten kann durch einen Kanülenreiz während der Larynxelevation verursacht sein und sollte endoskopisch abgeklärt werden. Nach Ausschluss einer Aspiration kann vor- übergehend eine unblockbare TK mit VK-A eingelegt werden. Regelmäßig wird das Anfärben der Zunge unter Absaugkontrolle zur Detektion einer Speichelaspiration durchgeführt.

\section{Endoskopische/bildgebende Schluckuntersuchung}

Bei ausreichender Wachheit und Kooperationsfähigkeit des Patienten wird eine orientierende klinische und bei klinischem Verdacht auf eine Aspiration - eine endoskopische Schluckuntersuchung mit unterschiedlichen Nahrungskonsistenzen durchgeführt (FEES [fiberoptic endoscopic evaluation of swallowing]). Nicht selten kann die endoskopische Schluckuntersuchung Auffälligkeiten der Atmung, der Schutzmechanismen und des Schluckens nachweisen. Schwellungen der ArytenoidKnorpel sind häufig durch eine einliegende Nasogastralsonde verursacht. Durch Intubationstraumen können narbige Veränderungen zu kopfhaltungsabhängigen Verengungen einer oder beider Sinus piriformes (was die Penetration von Nahrung oder Speichel bewirkt und durch Veränderung der Kopfhaltung behoben werden kann) und ein- oder beidseitige Stimmlippenbewe- 
gungsstörungen entstehen. Die bildgebende Diagnostik macht auch Fehllagen der TK oder eine Vorwölbung der Pars membranacea mit intermittierendem TK-Verschluss sichtbar. Nicht unerwähnt bleiben soll auch, dass der Einsatz bildgebender Verfahren gepaart mit unzureichender Kenntnis der pharyngealen und laryngealen Anatomie das Risiko falsch pathologischer Befunde birgt.

Nahrungskonsistenzen, die in der Untersuchung mit oder ohne Kompensationen einen ungestörten Schluckakt zeigen, sollen in der Folgezeit zum sicheren Trainieren des Schluckaktes und zur Verbesserung der Schluckphysiologie verabreicht werden.

\section{Nächste Schritte zur Dekanülierung}

Erfolgten die Entblockungsmaßnahmen zunächst in einer bestimmten Ausgangslage, so werden sie im Verlauf auch in anderen Ausgangslagen umgesetzt. Hier bietet sich vor allem die Zusammenarbeit mit den Angehörigen als sogenannte Co-Therapeuten an. Zeigen sich im Tagesverlauf keine erkennbaren Auffälligkeiten hinsichtlich Atmung und Speichelschluck, kann die Entblockungszeit sukzessive auf 24 Stunden ausgedehnt werden (Abb. 13). Wurde durch klinische und bildgebende Untersuchungsverfahren eine Speichelaspirationsgefahr weitestgehend ausgeschlossen, kann der vorübergehende Wechsel auf eine unblockbare TK erfolgen. Liegt keine fortbestehende Absaugnotwendigkeit vor, kann eine sofortige Dekanülierung vorgenommen werden.

\section{Supportive Maßnahmen}

Es bestehen mehrere Möglichkeiten, den Weg zur Dekanülierung positiv zu beeinflussen. So kann z.B. die Ausgangslage des Patienten verändert werden, um eine bessere Belüftung der Lunge zu erreichen und Atelektasen zu vermeiden bzw. um Sekret zu mobilisieren [61, 72]. Bereits in der Anfangsphase und die Behandlung stetig begleitend, sollten zur Pneumonieprophylaxe und Verbesserung der Atemphysiologie atemtherapeutische Maßnahmen eingesetzt werden. Nachweislich effektiv sind dabei: das sogenannte »bagging $[23,24]$ $\left(\mathrm{O}_{2}\right.$-Gabe über einen Beatmungsbeutel während der Inspiration des Patienten mit nachfolgendem therapieunterstützenden Husten) und oszillierende atemtherapeutische Geräte (Förderung der Sekretmobilisation über »Massage des Bronchialsystems« z. B. mit RC Cornet ${ }^{\circledR}$ oder Flutter VRP $1^{\circledR}$ ). Bei Patienten mit erschwerter Sekretmobilisation sollte eine sekretlösende Medikation, unterstützt durch einen sogenannten »Cough assist « mit mechanisch unterstützter In- und Exsufflation eingesetzt werden [71]. Zukünftig wird sich hier auch das Berufsbild des Atmungstherapeuten etablieren.
Interdisziplinäres Team

Durch die enge Zusammenarbeit in einem multidisziplinären Team mit gegenseitigem Respekt der verschiedenen Berufsgruppen lässt sich die Effektivität der Behandlung signifikant steigern $[25,27]$. Durch interne und externe Weiterbildungsmaßnahmen einschließlich Fachsupervisionen soll das Fachwissen kontinuierlich auf den aktuellen Forschungsstand angepasst werden. Aufgrund der unterschiedlichen Symptomkonstellation der Dysphagie bei tracheotomierten Patienten in der neurologischen Frührehabilitation ist ein individuelles ressourcenorientiertes Vorgehen unumgänglich. Innerhalb der Klinik etablierte und im Behandlungsteam konsentierte Behandlungspfade stützen die Wahl und den Zeitpunkt der Einleitung der einzelnen Behandlungsschritte. Bei beatmeten Patienten sind z.B. die Entblockungszeiten durch die Dauer der Spontanatemphasen limitiert. Hier ist das Führen eines Weaningprotokolls hilfreich, um die Entwöhnung von der Beatmung und der Trachealkanüle zu standardisieren und gleichzeitig parallel angebotene Übungsverfahren planbar durchführen zu können. Eine strenge Dokumentation mit Zeitangaben, Angaben zu Absaughäufigkeit, Sekretbeschaffenheit, Ausgangslage und Besonderheiten ist Grundlage der im Folgenden beschriebenen Behandlungsschritte. Das klinische Vorgehen bei der Entwöhnung von der TK ist schematisch in Abbildung 13 dargestellt.

Um unmittelbar Hilfe leisten zu können, sollte jeder Therapeut in das intratracheale Absaugen eingewiesen werden. Generell empfehlen wir eine Monitorpflicht mit zentraler Überwachung für alle tracheotomierten Patienten mit geblockter Trachealkanüle. Bei unauffälligem Verlauf sollen die beschriebenen Maßnahmen sukzessive zeitlich ausgedehnt werden. Anfangs ist eine fachtherapeutische (schlucktherapeutische) Begleitung notwendig, die in der Folgezeit im Delegationsverfahren an supervidiertes Fachpersonal (Pflegekräfte, Ergotherapeuten) übergeben werden kann.

\section{Fazit}

Die zunehmende Anzahl an intensiv- und überwachungspflichtigen Patienten in der NNFR stellt neue Herausforderungen an das interdisziplinäre Behandlungsteam. Die Entwöhnung von Beatmung und der TK hat sich mittlerweile zu einem Behandlungsschwerpunkt in der NNFR entwickelt. Das setzt die enge multidisziplinäre Zusammenarbeit voraus und fordert von allen beteiligten Professionen einen einheitlich hohen Kenntnisstand. Die Etablierung eines klinikinternen Behandlungsstandards ist für den raschen und lückenlosen Informationsaustausch zwischen den Berufsgruppen unerlässlich. Nur so können am individuellen Patienten Behandlungsmaßnahmen mit gleichbleibend hoher Qualität umgesetzt werden. Bei der Wahl der TK sollten 


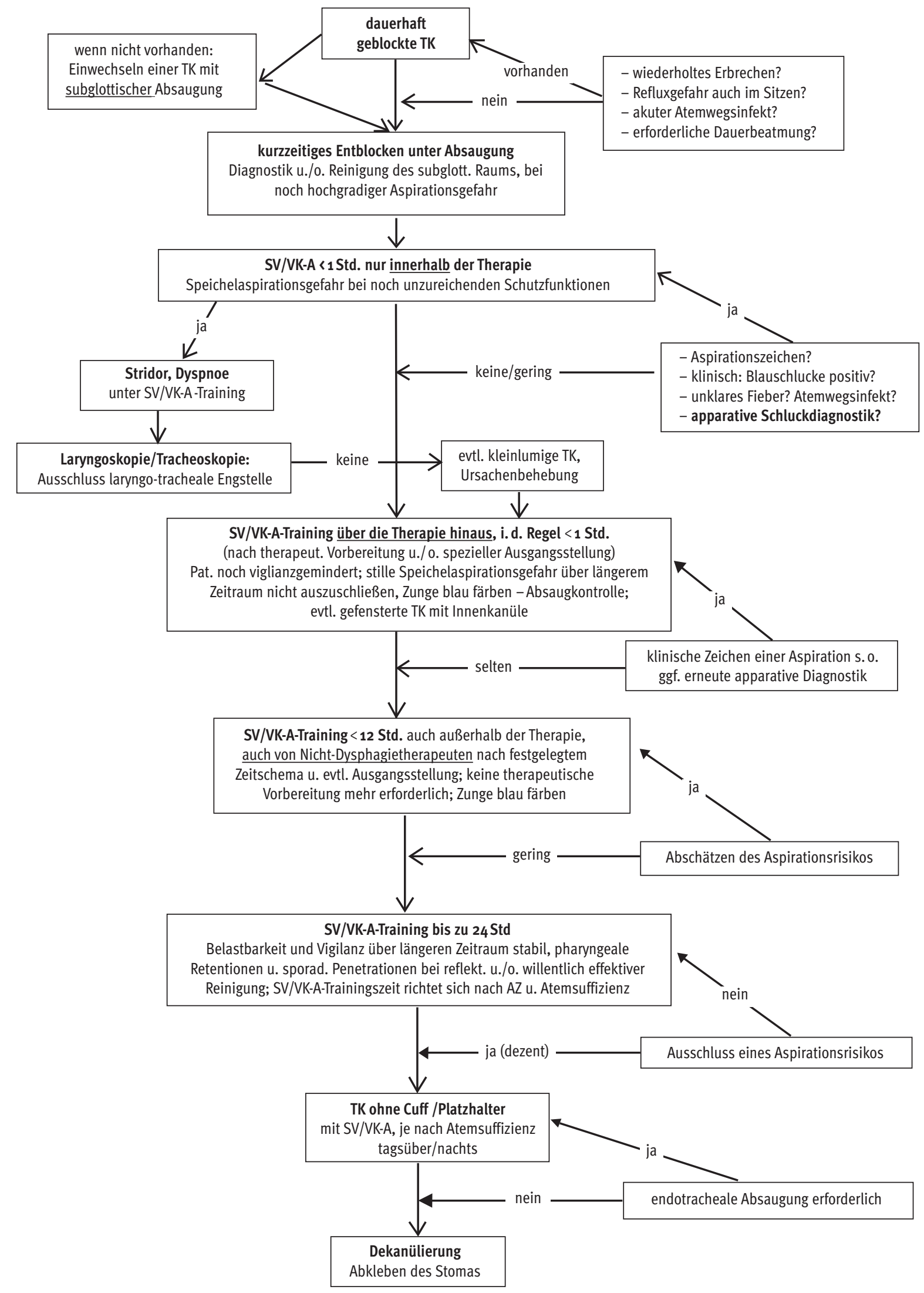

Abb. 13: Klinischer Behandlungspfad zur Dekanülierung 
neben den medizinischen Vorgaben vor allem auch die (im besten Fall mit dem Patienten formulierten) therapeutischen Ziele hinsichtlich Atmung und Schlucken berücksichtigt werden. Durch den frühzeitigen Einsatz eines SV/VK-A-Trainings und der damit verbundenen Luftstromumlenkung via Glottis kommt es nachweislich $\mathrm{zu}$ einer Verbesserung der pharyngolaryngealen Reizverarbeitung [31], einer besseren Belüftung und effektiveren Reinigung der Luftwege und der Lunge sowie einer Zunahme der spontanen Schluckaktivität [64]. Die Möglichkeit zur verbalen Kommunikation und erste Schritte in Richtung einer oralen Nahrungsaufnahme sind ein entscheidender Gewinn an Lebensqualität für den Patienten.

Fortschritte und Erfolge lassen sich durch ein Behandlungskontinuum mit täglichen Übungen und sukzessiver Verlängerung der TK-Entblockungszeiten unter Einhaltung präventiver Maßnahmen zur Vermeidung von Sekundärkomplikationen erzielen [25]. Neben den Behandlungsfortschritten sind auch Probleme und Komplikationen individuell zu dokumentieren, um unmittelbar darauf reagieren $\mathrm{zu}$ können. Um eine hochfrequente Behandlung zu garantieren, ist ein supervisorischer Behandlungsansatz unter Einbezug anderer Berufsgruppen (Pflege und Ergotherapie) sowie der Angehörigen sinnvoll. Dies erfordert ein Umdenken bei der gegenwärtig recht rigiden und berufsgruppenbezogenen Therapieplanung. Wie in vielen Bereichen der neurologischen Rehabilitation fehlt für die meisten bei der Entwöhnung von der Beatmung und der TK angewandten Therapieverfahren die wissenschaftliche Evidenz. Hier werden randomisierte kontrollierte Studien benötigt.

\section{Literatur}

1. Prävention der nosokomialen beatmungsassoziierten Pneumonie. Bundesgesundheitsblatt 2013; 56: 1578-1590.

2. Bertram M, Brandt T. Neurologische Frührehabilitation bei beatmeten Patienten mit ZNS-Störungen. Intensivmedizin up2date 2013; 9: 53-71.

3. Bögel I. Reduziert die Cuffdruckregelung die Inzidenz nosokomialer Pneumonie? Eine prospektive Studie. Dissertation Universität Hamburg 2001.

4. Bonanno PC. Swallowing dysfunction after tracheostomy. Ann Surg 1971; 174: 29-33.

5. Breatnach E, Abbott GC, Fraser RG. Dimensions of the normal human trachea. AJR Am J Roentgenol 1984; 142: 903-906.

6. Byhahn C, Wilke HJ, Halbig S, Lischke V, Westphal K. Percutaneous tracheostomy: ciaglia blue rhino versus the basic ciaglia technique of percutaneous dilational tracheostomy. Anesth Analg 2000; 91: 882-886.

7. Chadda K, Louis B, Benaissa L, Annane D, Gajdos P, Raphael JC, Lofaso F. Physiological effects of decannulation in tracheostomized patients. Intensive Care Med 2002; 28: $1761-1767$.

8. Charles MP, Kali A, Easow JM, Joseph NM, Ravishankar M, Srinivasan S, Kumar S, Umadevi S. Ventilator-associated pneumonia. Australas Med J 2014; 7: 334-344.

9. Cheung NH, Napolitano LM. Tracheostomy: epidemiology, indications, timing, technique, and outcomes. Respir Care 2014; 59: 895-915; discussion 916-899.
10. Ciaglia P, Firsching R, Syniec C. Elective percutaneous dilatational tracheostomy. A new simple bedside procedure; preliminary report. Chest 1985; 87: 715-719.

11. Clum SR, Rumbak MJ. Mortality and tracheotomy. Crit Care Med 2007; 35: 963-964.

12. D’Andrilli A, Venuta F, Rendina EA. Subglottic tracheal stenosis. J Thorac Dis 2016; 8: S140-147.

13. Damas P, Frippiat F, Ancion A, Canivet JL, Lambermont $\mathrm{B}$, Layios $\mathrm{N}$ et al. Ledoux D: Prevention of ventilator-associated pneumonia and ventilator-associated conditions: a randomized controlled trial with subglottic secretion suctioning. Crit Care Med 2015; 43: 22-30.

14. de Mestral C, Iqbal S, Fong N, LeBlanc J, Fata P, Razek T, Khwaja K. Impact of a specialized multidisciplinary tracheostomy team on tracheostomy care in critically ill patients. Can J Surg 2011; 54: 167-172.

15. Dembinski R, Rossaint R. Ventilator-associated pneumonia. Anaesthesist 2008; 57: 825-842.

16. Dettelbach MA, Gross RD, Mahlmann J, Eibling DE. Effect of the Passy-Muir Valve on aspiration in patients with tracheostomy. Head Neck 1995; 17: 297-302.

17. Ding R, Logemann JA. Swallow physiology in patients with trach cuff inflated or deflated: a retrospective study. Head Neck 2005; 27: 809-813.

18. Donzelli J, Brady S, Wesling M, Theisen M. Effects of the removal of the tracheotomy tube on swallowing during the fiberoptic endoscopic exam of the swallow (FEES). Dysphagia 2005; 20: 283-289.

19. Durbin CG, Jr. Techniques for performing tracheostomy. Respir Care 2005; 50: 488-496.

20. Epstein SK. Late complications of tracheostomy. Respir Care 2005; 50: 542-549.

21. Fantoni A, Ripamonti D. A non-derivative, non-surgical tracheostomy: the translaryngeal method. Intensive Care Med 1997; 23: 386-392.

22. Fernandez-Bussy S, Mahajan B, Folch E, Caviedes I, Guerrero J, Majid A. Tracheostomy Tube Placement: Early and Late Complications. J Bronchology Interv Pulmonol 2015; 22: 357-364.

23. Frank K, Frank U. Respiratory therapy (bagging, air stacking) for patients in early neurorehabilitation. Pneumologie 2011; 65: 314-319.

24. Frank U, Frank K, Zimmermann H. Effects of Respiratory Therapy (bagging) on Respiratory Function, Swallowing Frequency and Vigilance in Tracheotomized Patients in Early Neurorehabilitation. Pneumologie 2015; 69: 394-399.

25. Frank U, Mader M, Sticher H. Dysphagic patients with tracheotomies: a multidisciplinary approach to treatment and decannulation management. Dysphagia 2007; 22: 20-29.

26. Frova G, Quintel M. A new simple method for percutaneous tracheostomy: controlled rotating dilation. A preliminary report. Intensive Care Med 2002; 28: 299-303.

27. Garuti G, Reverberi C, Briganti A, Massobrio M, Lombardi F, Lusuardi M. Swallowing disorders in tracheostomised patients: a multidisciplinary/multiprofessional approach in decannulation protocols. Multidiscip Respir Med 2014; 9: 36.

28. Griggs WM, Worthley LI, Gilligan JE, Thomas PD, Myburg JA. A simple percutaneous tracheostomy technique. Surg Gynecol Obstet 1990; 170: 543-545.

29. Groves DS, Durbin CG, Jr. Tracheostomy in the critically ill: indications, timing and techniques. Curr Opin Crit Care 2007; 13: 90-97.

30. Hamishekar H, Shadvar K, Taghizadeh M, Golzari SE, Mojtahedzadeh M, Soleimanpour H, Mahmoodpoor A. Ventilator-associated pneumonia in patients admitted to intensive care units, using open or closed endotracheal suctioning. Anesth Pain Med 2014; 4: e21649.

31. Heidler M-D. Rehabilitation schwerer pharyngo-laryngotrachealer Sensibilitätsstörungen bei neurologischen Pati- 
enten mit geblockter Trachealkanüle. Neurologie \& Rehabilitation 2007; 13: 3-14.

32. Hess DR. Facilitating speech in the patient with a tracheostomy. Respir Care 2005; 50: 519-525.

33. Hess DR. Tracheostomy tubes and related appliances. Respir Care 2005; 50: 497-510.

34. Hess DR, Altobelli NP. Tracheostomy tubes. Respir Care 2014; 59: 956-971; discussion 971-953.

35. Hollaar V, Maarel-Wierink C, van der Putten GJ, Swart B, Baat C. Effect of daily application of a $0.05 \%$ chlorhexidine solution on the incidence of (aspiration) pneumonia in care home residents: design of a multicentre cluster randomised controlled clinical trial. BMJ Open 2015; 5: eoo7889.

36. Hudson JK, McDonald BJ, MacDonald JC, Ruel MA, Hudson CC. Impact of subglottic suctioning on the incidence of pneumonia after cardiac surgery: a retrospective observational study. J Cardiothorac Vasc Anesth 2015; 29: 59-63.

37. Husain T, Gatward JJ, Harris RD. Use of subglottic suction port to enable verbal communication in ventilator-dependent patients. Am J Respir Crit Care Med 2011; 184: 384.

38. Jeon YT, Hwang JW, Lim YJ, Lee SY, Woo KI, Park HP. Effect of tracheostomy timing on clinical outcome in neurosurgical patients: early versus late tracheostomy. J Neurosurg Anesthesiol 2014; 26: 22-26.

39. Johnson DC, Campbell SL, Rabkin JD. Tracheostomy tube manometry: evaluation of speaking valves, capping and need for downsizing. Clin Respir J 2009; 3: 8-14.

40. Jung SJ, Kim DY, Kim YW, Koh YW, Joo SY, Kim ES. Effect of decannulation on pharyngeal and laryngeal movement in post-stroke tracheostomized patients. Ann Rehabil Med 2012; 36: 356-364.

41. Kang JY, Choi KH, Yun GJ, Kim MY, Ryu JS. Does removal of tracheostomy affect dysphagia? A kinematic analysis. Dysphagia 2012; 27: 498-503.

42. Leder SB, Ross DA. Confirmation of no causal relationship between tracheotomy and aspiration status: a direct replication study. Dysphagia 2010; 25: 35-39.

43. Leder SB, Tarro JM, Burrell MI. Effect of occlusion of a tracheotomy tube on aspiration. Dysphagia 1996; 11: 254-258.

44. Ledl C, Mertl-Roetzer M. Tracheal and tracheostomal hypergranulation and related stenosis in long-term cannulated patients: does the tracheostomy procedure make a difference? Ann Otol Rhinol Laryngol 2009; 118: 876-880.

45. Lee YC, Kim TH, Lee JW, Oh IH, Eun YG. Comparison of complications in stroke subjects undergoing early versus standard tracheostomy. Respir Care 2015; 60: 651-657.

46. Lüdemann C WU. Messungen des auf die Trachealwand ausgeübten Druckes bei Beatmungskanülen mit herkömmlichen und einer neuartigen Blockungsmanschette. Prakt Anaesth 1972; 7: 217-226.

47. Maguire S, Haury F, Jew K. An in vitro comparison of tracheostomy tube cuffs. Med Devices (Auckl) 2015; 21: 185-192.

48. Niers N. Trachealkanülenmanagement: therapeutische Kriterien zur gezielten Auswahl passender Größen; LOGOS Interdisziplinär Jg. 20 Ausg. 12012 42-51. LOGOS Interdisziplinär 2012; 20: 42-51.

49. Nowak DA, Linden R, Riecker A. Schluckstörungen nach Schlaganfall. Pragmatisches Vorgehen in der Frührehabiliatation. Akt Neurol 2013; 40: 85-89.

50. Oehmichen F, Ketter G, Mertl-Rotzer M, Platz T, Puschendorf W, Rollnik JD, Schaupp M, Pohl M. Weaning from prolonged mechanical ventilation in neurological weaning units: an evaluation of the German Working Group for early Neurorehabilitation. Nervenarzt 2012; 83: 1300-1307.

51. Ortega-Suhrkamp E, von Wild KR. Standards of neurologicneurosurgical early rehabilitation--a concept of the study group neurological-neurosurgical early rehabilitation. Acta Neurochir Suppl 2002; 79: 11-19.
52. Passy V. Passy-Muir tracheostomy speaking valve. Otolaryngol Head Neck Surg 1986; 95: 247-248.

53. Passy V, Baydur A, Prentice W, Darnell-Neal R. Passy-Muir tracheostomy speaking valve on ventilator-dependent patients. Laryngoscope 1993; 103: 653-658.

54. Pauloski BR, Logemann JA, Colangelo LA, Rademaker AW, McConnel FM, Heiser MA et al., Esclamado R: Surgical variables affecting speech in treated patients with oral and oropharyngeal cancer. Laryngoscope 1998; 108: 908-916.

55. Pohl M, Berger K, Ketter G, Krusch C, Pause M, Puschendorf $\mathrm{W}$ et al., Mehrholz J. Langzeitverlauf von Patienten der neurologischen Rehabilitation Phase B: Ergebnisse der 6-Jahres-Nachuntersuchung einer Multizenterstudie. Nervenarzt 2011; 82: 753-763.

56. Ponfick M, Linden R, Nowak DA. Dysphagia--a common, transient symptom in critical illness polyneuropathy: a fiberoptic endoscopic evaluation of swallowing study* Crit Care Med 2015; 43: 365-372.

57. Ponfick M, Wiederer R, Bosl K, Neumann G, Ludemann Podubecka J, Gdynia HJ, Nowak DA. The influence of weaning duration on rehabilitative outcome in early neurological rehabilitation. NeuroRehabilitation 2014; 34: 493-498.

58. Prigent $H$, Lejaille $M$, Terzi $N$, Annane D, Figere $M$, Orlikowski D, Lofaso F. Effect of a tracheostomy speaking valve on breathing-swallowing interaction. Intensive Care Med 2012; 38: 85-90.

59. Rumbak MJ, Newton M, Truncale T, Schwartz SW, Adams JW, Hazard PB. A prospective, randomized, study comparing early percutaneous dilational tracheotomy to prolonged translaryngeal intubation (delayed tracheotomy) in critically ill medical patients. Crit Care Med 2004; 32: 1689-1694.

6o. Schnell R. Sprechventil für Tracheostomiekanüle. Google Patents; 2014.

61. Schonhofer B, Geiseler J, Dellweg D, Moerer O, Barchfeld T, Fuchs $\mathrm{H}$ et al., Windisch W. S2k-Guideline »Prolonged Weaning«. Pneumologie 2015; 69: 595-607.

62. Schulz-Stübner S, Forster E, Kniehl E, Kehl F: Städtisches Klinikum Karlsruhe Mundpflege: Veränderungen des Keimspektrums in Surveillancekulturen PO-2.4.6 Prävention der beatmungsassoziierten Pneumonie durch Octenidin Anästh Intensivmed 2010; 51: 178.

63. Schulz-Stübner S, Kniehl E, Sitzmann F: Die Rolle der Mundpflege in der Prävention beatmungsassoziierter Pneumonien. Krankenhaushygiene up2date 2010; 5: 177-190.

64. Seidl RO, Nusser-Müller-Busch R, Ernst A. The influence of tracheotomy tubes on the swallowing frequency in neurogenic dysphagia. Otolaryngol Head Neck Surg 2005; 132: 484-486.

65. Stonecypher K. Ventilator-associated pneumonia: the importance of oral care in intubated adults. Crit Care Nurse 2010; 33: 339-347.

66. Storm B, Dybwik K, Nielsen EW. Late complications after percutaneous tracheostomy and oral intubation: Evaluation of 1,628 procedures. Laryngoscope 2015

67. Suiter DM, McCullough GH, Powell PW. Effects of cuff deflation and one-way tracheostomy speaking valve placement on swallow physiology. Dysphagia 2003; 18: 284-292.

68. Sutt AL, Cornwell P, Mullany D, Kinneally T, Fraser JF The use of tracheostomy speaking valves in mechanically ventilated patients results in improved communication and does not prolong ventilation time in cardiothoracic intensive care unit patients. J Crit Care 2015; 30: 491-494.

69. Terk AR, Leder SB, Burrell MI. Hyoid bone and laryngeal movement dependent upon presence of a tracheotomy tube. Dysphagia 2007; 22: 89-93.

70. Terragni PP, Antonelli M, Fumagalli R. Early vs late tracheotomy for prevention of pneumonia in mechanically ventilated adult ICU patients: a randomized controlled trial. JAMA 2010; 303: 1483-1489. 

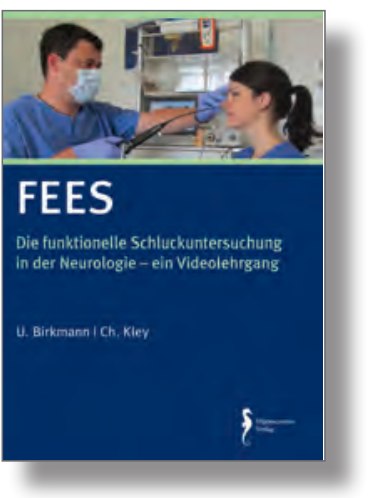

U. Birkmann, Ch. Kley

\section{Die funktionelle Schluckuntersuchung in der Neurologie (FEES) - ein Videolehrgang}

\section{Ein Videolehrgang}

Hippocampus Verlag,

Bad Honnef 2015

broschiert, 56 S., zahlr. Abb.

$€$ 34,90; ISBN 978-3-955441-13-5

Die Fiberendoskopische Evaluation des Schluckens (FEES) mit einem flexiblen Laryngoskop hat sich seit der Erstbeschreibung durch Susan Langmore zu einer Standarduntersuchung für Patienten mit Schluckstörungen entwickelt. Die Autoren stellen in einem ersten Kapitel zunächst die Technik der FEES mit den wichtigsten Informationen zum Untersuchungsgang vor. Sie zeigen Normalbefunde und die durch therapeutische Manöver erzielbaren Effekte auf. Anschließend erläutern sie an einzelnen Erkrankungen mit jeweiligen Fallbeispielen die typischen FEES-Befunde bei Schlaganfall, Morbus Parkinson und Amyotropher Lateralsklerose (ALS) und wiederum die Einflussmöglichkeiten, die dem Schlucktherapeuten zur Verfügung stehen.

Die zugehörige DVD enthält knapp 50 kommentierte Filmbeispiele mit typischen FEES-Befunden bei diesen Erkrankungen.

Inkl. DVD mit zahlreichen Videobeispielen!
71. Toussaint M. The use of mechanical insufflation-exsufflation via artificial airways. Respir Care 2011; 56: 1217-1219.

72. van Gestel A, Teschler H. Physiotherapie bei chronischen Atemwegs- und Lungenerkrankungen. Springer Verlag 2014: 277-304.

73. Villwock JA JK. Outcomes of early versus late tracheostomy: 2008-2010. Laryngoscope 2014; 124: 1801-1806.

74. Villwock JA, Villwock MR, Deshaies EM. Tracheostomy timing affects stroke recovery. J Stroke Cerebrovasc Dis 2014; 23: 1069-1072.
Interessenvermerk

Die Autoren geben an, dass kein Interessenkonflikt vorliegt.

Korrespondenzadresse:

Prof. Dr. Dennis A. Nowak

HELIOS Klinik Kipfenberg GmbH

Konrad-Regler-Straße 1

85110 Kipfenberg

E-Mail: dennis.nowak@helios-kliniken.de 